OPEN ACCESS

Edited by:

Chunbao Li,

Nanjing Agricultural University, China

Reviewed by: Ilkin Yucel Sengun,

Ege University, Turkey

Zhi-Qiang Xiong,

University of Shanghai for

Science and Technology, China

${ }^{*}$ Correspondence:

Xinping Lin

yingchaer@163.com

Beiwei Zhu

zhubeiwei@163.com

Specialty section: This article was submitted to

Food Microbiology,

a section of the journal

Frontiers in Microbiology

Received: 31 August 2018 Accepted: 20 November 2018 Published: 17 December 2018

Citation:

Bao R, Liu S, Ji C, Liang H, Yang S, Yan $X$, Zhou Y, Lin $X$ and Zhu $B$ (2018) Shortening Fermentation Period and Quality Improvement of Fermented Fish, Chouguiyu, by Co-inoculation of Lactococcus lactis M10 and Weissella cibaria M3. Front. Microbiol. 9:3003. doi: 10.3389/fmicb.2018.03003

\section{Shortening Fermentation Period and Quality Improvement of Fermented Fish, Chouguiyu, by Co-inoculation of Lactococcus lactis M10 and Weissella cibaria M3}

\author{
Ruiqi Bao', Sasa Liu', Chaofan Ji', Huipeng Liang', Song Yang², Xiaoming Yan², \\ Yingqin Zhou' ${ }^{2}$ Xinping Lin ${ }^{1 *}$ and Beiwei Zhu ${ }^{\text {t* }}$ \\ ${ }^{1}$ National Engineering Research Center of Seafood, Dalian Polytechnic University, Dalian, China, ${ }^{2}$ Institute of \\ Agro-products Processing, Anhui Academy of Agricultural Science, Hefei, China
}

Chouguiyu, a Chinese traditional fermented fish, is famous for its uniquely strong odor and desirable taste. However, traditional spontaneous fermentation often resulted in contamination and unstable quality of products. In this study, individual or conjunctive inoculation of two indigenous lactic acid bacteria ( $L A B)$, Lactococcus lactis M10 and Weissella cibaria M3, was tested for their effect on improving Chouguiyu's quality. It was shown that inoculation would not affect the system's pH, while increased the total bacteria count and lactic acid bacteria amounts. Matrix-assisted laser desorption/ionization time-of-flight mass (MALDI-TOF) analysis results revealed that Lactoc. lactis M10 and $W$. cibaria M3 could quickly occupy a dominant position in the ecosystem, and Lactoc. lactis M10 played an important role in the control of spoilage bacteria. Volatile basic nitrogen (TVB-N), thiobarbituric acid reactive substances (TBARS), and biogenic amines results also showed that Lactoc. lactis M10 had a positive effect on improving the product's quality. Co-inoculation of Lactoc. lactis M10 and W. cibaria M3 could promote the formation of flavor according to the E-nose and gas chromatography-mass spectrometer (GC-MS) analyses, especially for the aroma-active and key volatile compounds. PCA plots of E-nose and hierarchical clustering analysis of GC-MS profiles revealed that the co-inoculation sample at the fifth day (LW5) was the most similar to the natural fermentation sample at the seventh day (C7). The overall acceptance of LW5 was also the closest to that of $\mathrm{C} 7$ in sensory evaluation. In conclusion, mixed starter culture was shown to have a good effect on improving product quality and enhancing flavor with fermentation time shortened by $29 \%$.

Keywords: fermented fish, starter cultures, flavor, E-nose, GC-MS, MALDI TOF-MS, quality

\section{INTRODUCTION}

Siniperca chuatsi is a widely cultivated fish in China, and is of great commercial value (Yang et al., 2016). However, due to the rapid deterioration of fresh Si. chuatsi meat, fermentation process was developed and resulted in a well-known fish product called Chouguiyu, also known as stinky mandarin fish. Similar to a traditional Chinese soybean food-stinky tofu, this special fermented fish has a 
uniquely strong, smelly odor but desirable taste, and is widely welcome by the majority of consumers in China. Traditionally, the fresh fish Si. chuatsi was killed and cured with spices and salts, and then stored at $12 \pm 4^{\circ} \mathrm{C}$ for 7-12 days in jars with heavy stones on the top layer of the fish, and the jars were sealed with covers (Yang et al., 2017).

It was known that a spontaneous fermentation process was characterized by a complex community of microorganisms, which was important for the degradation of substrates, such as protein and carbohydrate, and gradual formation of fermented flavors (Thierry et al., 2016). In the spontaneous fermentation process, it took a long time for the system to form the dominant microbial community. This has become the bottleneck for industrial production of most traditional fermented foods. Besides, the natural microbial community was easily influenced and contaminated by the environmental conditions, such as variable temperature, spoilage, and pathogen bacteria. Manufacture factors, such as moisture or temperature (Yang et al., 2017), had been investigated for their effect on the Chouguiyu's quality, while it was still difficult to produce fermented fish with stable quality. The diversity of lactic acid bacteria involved in Chouguiyu fermentation had been investigated by Dai et al. (2013), and Lactobacillus sakei was found as the dominant species during the fermentation process. However, little research has been done on selecting starter culture to improve the Chouguiyu's quality.

Inoculation of starter culture is a common strategy to improve the quality of fermented foods (de Almeida et al., 2018; Xu et al., 2018). Compared with spontaneous fermentation, inoculation of starter culture into fermented foods possesses more advantages, such as shortening fermentation time, delaying spoilage, promotion of flavor, and improving product's quality. Lactic acid bacteria (LAB), known as the most popular starter culture, have been widely applied in various fermentation process of foods, such as meat (Kargozari et al., 2014), fish (Zeng et al., 2013; Gao et al., 2018), and cheese (Ruggirello et al., 2018) manufacture. It had been reported that indigenous LAB originated from the fermented fish Suanyu were able to quickly adapt to the ecology, and inhibit the growth of spoilage bacteria and pathogens as well as the increase of thiobarbituric acid reactive substances (TBARS) and volatile basic nitrogen (TVB-N) (Zeng et al., 2013). Gao et al. (2018) found that the inoculation of Lactob. plantarum could accelerate the formation of the flavors, especially the esterification and alcoholysis biosynthesis pathway in fermented fish. Biogenic amines accumulation could also be reduced by Lactob. plantarum during the fermentation of silver carp sausage (Zhang et al., 2013). According to previous study on Chouguiyu (Ji et al., 2017), LAB were discovered in fermented fish and were found to have impact on enhancing carbon metabolic pathway, which might have a function in the flavor formation.

In this study, the microbiological, physicochemical, volatile, and sensory properties of Chouguiyu with single starter culture (Lactococcus lactis M10, Weissella cibaria M3), mixed starter culture (Lactoc. lactis M10 and W. cibaria M3), and without starter culture (the control) were evaluated. The purpose of this study was to enhance the quality of product and shorten fermentation time by inoculation of starter cultures.

\section{MATERIALS AND METHODS}

\section{Starter Cultures}

In the beginning of doing this experiment, nine isolated LAB from Chouguiyu were used to inoculate the fish and the sensory evaluation was performed. Two bacteria, Lactoc. lactis M10 (CGMCC 16612) and W. cibaria M3 (CGMCC 16611), showed higher sensory scores than the other strains (unpublished results). These two strains were identified by $16 \mathrm{~S}$ rDNA analysis with primers of 27F (5'-AGAGTTTGATCCTGGCTCAG-3') and 1492R (5'-GGTTACCTTGTTACGACTT-3'). Lactoc. lactis M10 and $W$. cibaria M3 were cultured in MRS broth (Qingdao Hope Bio-Technology Co., Ltd) at $37^{\circ} \mathrm{C}$ for $18 \mathrm{~h}$. Cell pellets were harvested by centrifuging at $10,000 \times g$ for $2 \mathrm{~min}$ at $4^{\circ} \mathrm{C}$. Subsequently, they were washed twice with saline water $(0.9 \%$ $\mathrm{NaCl}$ ) and re-suspended in 15-ml sterile water before use.

\section{Chouguiyu Sample Preparation}

Fresh Si. chuatsi (average weight $600 \pm 100 \mathrm{~g}$ ) were purchased from Changxing market in Dalian, China. Fish were stored in ice within $24 \mathrm{~h}$ after being caught from the fish grounds. Then, the fish were gutted and prepared according to the traditional techniques by soaking the fish in salt $(6 \% \mathrm{w} / \mathrm{v})$ water with fennel $(0.02 \% \mathrm{w} / \mathrm{w})$, cumin $(0.04 \% \mathrm{w} / \mathrm{w})$, anise $(0.06 \% \mathrm{w} / \mathrm{w})$, Chinese prickly ash $(0.03 \%$ $\mathrm{w} / \mathrm{w})$, chilli powder $(0.002 \% \mathrm{w} / \mathrm{w})$, ginger $(0.6 \% \mathrm{w} / \mathrm{w})$, and shallot $(1.0 \% \mathrm{w} / \mathrm{w})$. Then, the fish and salt water were divided into four batches and inoculated with different starter cultures, including $\mathrm{L}$ (Lactoc. lactis M10), W (W. cibaria M3), LW (Lactoc. lactis M10 and $W$. cibaria $M 3,1: 1)$, and a batch without any starter $(C)$ as the control. Each starter culture ( $7 \log \mathrm{cfu} \mathrm{m}^{-1}$ ) was inoculated into the curing liquid and thoroughly mixed. The fish were covered with heavy bags (average weight of $7 \mathrm{~kg}$ for each fish). Fish containers were covered and stored at $12 \pm 1^{\circ} \mathrm{C}$ for $5-7$ days. Samples were collected on days 1, 3, and 5 for microbiological, physicochemical, and volatile analyses. Sensory evaluation was carried out at the end of the process.

\section{Microbiological Analysis}

Liquid sample of volume $1 \mathrm{~mL}$ was aseptically transferred to a sterile test tube with $9 \mathrm{ml}$ of sterile normal saline $(0.9 \% \mathrm{NaCl})$. Serial 10-fold dilutions were performed with the same normal saline, and $0.2 \mathrm{ml}$ of each dilution was inoculated in different growth media for microbial counting. LAB on deMan, Rogosa, and Sharpe (MRS, QingDao Hope Bio-technology Co., Ltd) agar was incubated at $37^{\circ} \mathrm{C}$ for $36-48 \mathrm{~h}$ and plate count agar (QingDao Hope Bio-technology Co., Ltd) for total bacteria count was incubated at $37^{\circ} \mathrm{C}$ for $36-48 \mathrm{~h}$ (Essid and Hassouna, 2013). The results were shown as colony-forming units per milliliter (cfu/ $\mathrm{ml}$ ). At the end of cultivation, the fresh colony from MRS and plate count agar plates was used to identify the microbial diversity with matrix-assisted laser desorption/ionization time-of-flight mass spectrometry (MALDI TOF-MS) (Bruker, Autoflex, Germany) according to the previous reports (da Cruz Pedrozo Miguel et al., 2017; El Hamzaoui et al., 2018). Briefly, a colony was scraped from the plate and placed on a steel target plate. Then, $1 \mu \mathrm{l}$ of $70 \%$ formic acid was directly covered onto the 
colony. After air drying, $1 \mu \mathrm{l}$ of matrix solution ( $50 \mu \mathrm{l}$ acetonitrile, $47.5 \mu \mathrm{l}$ water, $2.5 \mu \mathrm{l}$ trifluoroacetic acid, and $1 \mathrm{mg} \alpha$-cyano-4hydroxycinnamic acid) was added to each spot and air dried. This colony was analyzed by MALDI TOF-MS. Assays were carried out on Microflex LT bench-top mass spectrometer with FlexControl 3.0 software.

\section{Determination of $\mathrm{pH}$, TVB-N, and TBARS}

The $\mathrm{pH}$ value of fermentation liquid was measured by a $\mathrm{pH}$ meter (MettlerToledo Instruments Co., Ltd., Shanghai, China). TVB-N content was determined by Cobb's method (Cobb et al., 1973). The TVB-N concentration was expressed as $\mathrm{mg} / 100 \mathrm{~g}$ fish. TBARS assay was carried out according to John's report (John et al., 2005) and TBARS concentration was expressed as $\mathrm{mg} / \mathrm{kg}$.

\section{Biogenic Amine Determination}

Biogenic amine contents of Chouguiyu samples at the end of fermentation were analyzed according to a previous report (Zhang et al., 2013). Biogenic amines were extracted with $0.4 \mathrm{M}$ perchloric acid and then the extractions were derivatived with dansyl chloride according to Mah's report (Mah et al., 2002). Hypersil ODS C18, $5 \mathrm{~mm}, 4.6 \times 200 \mathrm{~mm}$ column (Thermo, Bellefonte, PA, USA) was used to separate the derivatives with temperature of $35^{\circ} \mathrm{C}$ and flow rate of $1 \mathrm{ml} / \mathrm{min}$. Mobile phase $\mathrm{A}$ ( $0.01 \mathrm{M}$ ammonium acetate) and mobile phase B (90\% acetonitrile) were used in a gradient elution program. The program was started at $45 \% \mathrm{~A}$ and $55 \% \mathrm{~B}$, and then raised to $95 \% \mathrm{~B}$ within $25 \mathrm{~min}$ and held for $10 \mathrm{~min}$. Biogenic amines were detected at $254 \mathrm{~nm}$ and expressed in $\mathrm{mg} / \mathrm{kg}$.

\section{Volatile Compounds Analysis by E-Nose and GC-MS}

The volatile compounds were analyzed by the E-nose (PEN3, Airsense, Germany) and their concentrations were determined by solid-phase gas chromatography-mass spectrometer (GC-MS) equipped with micro-extraction (HS-SPME) according to Li's study (Li et al., 2013). For electronic nose detection, the samples were taken in $40-\mathrm{ml}$ vials and heated at $60^{\circ} \mathrm{C}$ for $30 \mathrm{~min}$. The measurement phase was set for $70 \mathrm{~s}$ until the sensors reached stable values. The performance characteristics for the 10 different metal oxide sensors are listed in Table S2. For SPME, a fiber with $50 / 30 \mu \mathrm{m}$ divinylbenzene/carboxen/polydimethylsiloxane (DVB/CAR/PDMS; Supelco Inc., Bellefonte, AL, USA) was used to extract volatile compounds by stirring at $60^{\circ} \mathrm{C}$ for $70 \mathrm{~min}$. The adsorbent volatiles were thermally desorbed at $260^{\circ} \mathrm{C}$ for $5 \mathrm{~min}$. Volatiles were separated by a capillary column HP-5MS $(30 \mathrm{~m} \times 250 \mu \mathrm{m} \times 0.25 \mu \mathrm{m}$, Agilent Technologies Inc., California, USA). Helium was used as a carrier and its flow rate was $1.5 \mathrm{ml} /$ $\min$. The initial temperature was $30^{\circ} \mathrm{C}$ for $5 \mathrm{~min}$; thereafter, the column temperature was raised at a rate of $3^{\circ} \mathrm{C} / \mathrm{min}$ to $50^{\circ} \mathrm{C}$ (held $3 \mathrm{~min}$ ), then $5^{\circ} \mathrm{C} / \mathrm{min}$ to $150^{\circ} \mathrm{C}$, and finally $20^{\circ} \mathrm{C} / \mathrm{min}$ to $250^{\circ} \mathrm{C}$ (held $5 \mathrm{~min}$ ). Retention indices (RIs) were calculated by determining retention time of a mixture of n-alkanes $\left(\mathrm{C}_{5}-\mathrm{C}_{20}\right)$ under same GC conditions with samples. Volatiles were semiquantified by 2,4,6-trimethylpyridine as an internal standard and identified based on comparison of mass spectral data with GC-MS library (NIST11, Agilent Technologies, Inc., Santa Clara, CA).

\section{Sensory Analysis}

Samples were evaluated by sensory evaluation to assess the differences between control group (fermented for 7 days) and samples inoculated with starter cultures (fermented for 5 days) by a taste panel composed of 10-member food professional students. A preparatory session was held prior to the evaluation to ensure that each panelist could thoroughly discuss and clarify Chouguiyu's attributes. A 10-point hedonic scale, in which a score of 1 equals dislike extremely, 5 equals neither like nor dislike, and 10 equals like extremely, was used for evaluation. The evaluations were performed in individual booths. The panelists were asked to evaluate the appearance, aroma, taste, tactile texture, and overall acceptance. The results of sensory scores were calculated as the mean value of panelists' scores.

\section{Statistical Analysis}

Statistical analysis was carried out using SPSS (version 22.0, IBM, USA). The results were expressed as mean \pm SD and analysis of variance (ANOVA) was used. When $p<0.05$, the variation was considered to change the dependent variables significantly. Analyses of data collected from the E-nose were performed by WinMuster (Airsense, Germany). Principal component analysis (PCA) was used to investigate the sensor data between the control and samples inoculated with starter cultures using Canoco for Windows 4.5 software (Wageningen UR, Netherlands). R (version 3.4.4) was used to design the thermography and cluster analysis.

\section{RESULTS AND DISCUSSION}

\section{Changes of pH, Total Bacteria, and Lactic Acid Bacteria During the Fermentation}

The initial $\mathrm{pH}$ ranged from 6.81 to 6.97 in the control and inoculated samples (Table 1). During the fermentation process, $\mathrm{pH}$ values decreased slightly and fluctuated between 6.47 and 6.58 in all samples. It was also observed in traditional Korean fish sauce saeujeot (Lee et al., 2014), in which pH gradually dropped to 6.9-7.0 at the first 10 days. Zeng et al. (2016) also reported the $\mathrm{pH}$ value was about 6.0 at the first 10 days' fermentation in Suanyu, a traditional fermented fish in China, with LAB as starter culture. Fermented foods usually have a low $\mathrm{pH}$, such as salami (Essid and Hassouna, 2013), whose $\mathrm{pH}$ values varied between 4.2 and 5.3. However, the $\mathrm{pH}$ values of stinky mandarin fish were higher than other fermented foods, mainly due to its shorter fermentation time. There was no significant difference between the inoculated groups and the control one, indicating that inoculation of starter culture did not have a significant effect on $\mathrm{pH}$ values.

For microorganisms, the initial amount of total bacteria in the control group was $4.53 \mathrm{log} \mathrm{cfu} / \mathrm{ml}$ (Table 1), which gradually increased to $5.51 \log \mathrm{cfu} / \mathrm{ml}$ at the end of fermentation. The initial amounts of total bacteria in L, W, and LW groups' samples ranged from 5.33 to $6.18 \log \mathrm{cfu} / \mathrm{ml}$, which was higher than that of the control due to the inoculation of LAB. At the end of fermentation, no significant difference was observed between the total bacteria amount of the control and batches with starter, except for the L group. The same tendency was observed in LAB counting (Table 1). 
TABLE 1 | Changes of $\mathrm{pH}$, total bacteria count, and lactic acid bacteria during the fermentation.

\begin{tabular}{|c|c|c|c|c|c|c|c|c|c|}
\hline \multirow{2}{*}{ Samples $^{A}$} & \multicolumn{3}{|c|}{$\mathrm{pH}$} & \multicolumn{3}{|c|}{ Total bacteria count (log cfu/ml) } & \multicolumn{3}{|c|}{ Lactic acid bacteria (log cfu/ml) } \\
\hline & 1D & 3D & 5D & 1D & 3D & 5D & 1D & 3D & 5D \\
\hline C & $6.81 \pm 0.05^{\mathrm{a} 1}$ & $6.48 \pm 0.07^{\mathrm{ab} 1}$ & $6.58 \pm 0.12^{\mathrm{a} 1}$ & $4.53 \pm 0.01^{\mathrm{a} 1}$ & $4.82 \pm 0.07^{\mathrm{a} 1}$ & $5.51 \pm 0.19^{\mathrm{a} 2}$ & $4.53 \pm 0.16^{\mathrm{a} 2}$ & $4.02 \pm 0.04^{\mathrm{a} 1}$ & $4.61 \pm 0.10^{\mathrm{a} 2}$ \\
\hline $\mathrm{L}$ & $6.83 \pm 0.04^{\mathrm{a} 2}$ & $6.50 \pm 0.03^{\mathrm{a} 1}$ & $6.47 \pm 0.05^{\mathrm{a} 1}$ & $6.18 \pm 0.03^{\mathrm{d} 1}$ & $6.53 \pm 0.03^{\mathrm{d} 2}$ & $6.62 \pm 0.07^{\mathrm{b} 2}$ & $6.02 \pm 0.07^{\mathrm{c} 1}$ & $6.52 \pm 0.02^{\mathrm{c} 2}$ & $6.56 \pm 0.11^{\mathrm{c} 2}$ \\
\hline W & $6.87 \pm 0.01^{\mathrm{a} 3}$ & $6.48 \pm 0.01^{\mathrm{a} 2}$ & $6.57 \pm 0.03^{\mathrm{a} 1}$ & $5.33 \pm 0.06^{b 1}$ & $5.47 \pm 0.06^{\mathrm{b} 1}$ & $5.56 \pm 0.08^{\mathrm{a} 2}$ & $6.14 \pm 0.05^{\mathrm{c} 1}$ & $5.97 \pm 0.27^{\mathrm{b} 1}$ & $5.90 \pm 0.06^{b 1}$ \\
\hline LW & $6.97 \pm 0.02^{\mathrm{b} 3}$ & $6.64 \pm 0.02^{\mathrm{b} 2}$ & $6.49 \pm 0.04^{\mathrm{a} 1}$ & $5.70 \pm 0.02^{c 1}$ & $5.78 \pm 0.03^{\mathrm{c} 1}$ & $5.83 \pm 0.08^{\mathrm{a} 1}$ & $5.54 \pm 0.11^{\mathrm{b} 1}$ & $5.81 \pm 0.08^{\mathrm{b} 2}$ & $6.00 \pm 0.01^{\mathrm{b} 2}$ \\
\hline
\end{tabular}

Values with unlike superscript letters $(a-c)$ in the same column are significantly different $(p<0.05)$. Values with unlike superscript letters (1-3) in the same row of between the fermentation process are significantly different $(p<0.05) .{ }^{A} C, L, 5$, and LW represented fish fermented without starter, with Lactoc. lactis M10, with W. cibaria M3, and with both of the bacteria for 5 days.

The number of LAB in the L group was higher than that of the other three batches, which indicated that Lactoc. lactis M10 could grow well in this system. The LAB amounts in $\mathrm{W}$ and LW groups were also higher than that of the control, which suggested the inoculated strains could adapt well to the fermentation conditions and became the dominant bacteria in the microbiota community of stinky mandarin fish. It had been shown that LAB, as the dominant bacteria, played an important role in fermented foods by utilizing carbohydrates and proteins to produce lactic acid and bacteriocins, and could improve the flavors of products and inhibit the growth of spoilage bacteria, which had a positive impact on the quality of products (Zhang et al., 2013; Gao et al., 2018). Co-inoculation of LAB greatly increased their numbers and shortened the time for LAB occupying the dominant position in Chouguiyu.

\section{Bacterial Community Succession During the Fermentation}

In order to further understand the changes of bacterial succession during the fermentation process, MALDI-TOF was used to identify the bacterial compositions on plate count agar (Figure 1A) and MRS plates (Figure 1B). The bacterial diversity in the control group is more complex than that in the other three groups. This resulted from various microorganisms attached in the surface of fish body, such as Aeromonas (Montes et al., 1999), which were facultative anaerobic bacteria and occurred ubiquitously and autochthonously in aquatic environments. The amounts of Lactoc. lactis M10 and W. cibaria M3 increased from 4.76 to $20.93 \%$ during the fermentation on the plate count agar plate. What is more, it was observed that the increase of Lactoc. lactis M10 was greater than that of W. cibaria M3 on both plate count agar and MRS plates in the control group, implying that these two strains may have competitive relationships in the fermentation system.

For the group with starter cultures, it could be seen that the dominant bacteria were the starter culture they inoculated, Lactoc. lactis M10 and W. cibaria M3. For sample L, Lactoc. lactis became the only dominant bacteria on either plate count agar or MRS plates throughout the fermentation process. It was reported that Lactoc. lactis was capable of producing bacteriocin (Saelao et al., 2018), such as nisin, which could suppress the spoilage or pathogen bacteria. Ho et al. found that in the presence of Lactoc. lactis KTH0-1S, pathogenic bacteria, such as L. monocytogenes decreased significantly in cheese (Ho et al., 2018). For the W group, strains such as Aeromonas were detected in addition to W. cibaria M3. A. salmonicida (Park et al., 2017) and Streptococcus parauberis
(Fernandez-No et al., 2012) were reported as the pathogenic bacteria in aquatic products and were commonly found in food spoilage. The reason for more spoilage bacteria found in group $\mathrm{W}$ may be due to the weak ability of $W$. cibaria M3 to produce bacteriocin and its limited ability to inhibit other microorganisms. It is reported that not all W. cibaria (Li et al., 2017) strains could produce bacteriocin, but the production capacity was quite different between strains (Woraprayote et al., 2015). In addition, Lactoc. lactis M10 increased with the decrease of W. cibaria M3 during the fermentation (Figures 1A,B), either on the plate count agar or MRS plates, indicating that there was a competitive relationship between the two strains, which was the same as that in the control group. Romi also observed in fermented bamboo shoots (Romi et al., 2015) that W. cibaria was gradually decreased or even disappeared with the extension of fermentation time, while Lactoc. lactis subsp. could be detected during the whole fermentation period. Therefore, Lactoc. lactis might have a competitive relationship with $W$. cibaria by occupying a dominant position in the ecosystem, and played an important role in the control of spoilage bacteria and improvement of the product quality.

\section{Changes of TVB-N, TBARS, and Biogenic Amines During the Fermentation}

The initial TVB-N contents were in the range of 20.32$22.90 \mathrm{mg} / 100 \mathrm{~g}$. Then, TVB-N values significantly increased and reached $49.49 \mathrm{mg} / 100 \mathrm{~g}$ at the fifth day in the control group. TVB-N concentrations in the $\mathrm{W}$ and $\mathrm{LW}$ groups were $52.68 \mathrm{mg} / 100 \mathrm{~g}$ and $47.49 \mathrm{mg} / 100 \mathrm{~g}$, respectively, and showed no significant difference with the control group. In contrast, TVB-N content $(34.17 \mathrm{mg} / 100 \mathrm{~g})$ was greatly decreased in the fish inoculated with Lactoc. lactis M10. This result was in accordance with Hu's (Hu et al., 2008) and Yin's studies (Yin et al., 2002), who both reported that LAB inoculation could inhibit the accumulation of TVB-N by producing lactic acid and bacteriocins in fish or meat fermentation.

Si. chuatsi is a kind of fish containing highly unsaturated lipids, which are easily susceptible to oxidation and resulted in a rancid smell and taste. TBARS is often used as an indicator for the degree of lipid oxidation. It has been reported that fish is of good quality when the TBARS content is below $5 \mathrm{mg} / \mathrm{kg}$ (Zeng et al., 2013). In our study, the initial TBARS contents were $0.03-0.72 \mathrm{mg}$ of MDA/ $\mathrm{kg}$. At the end of 5 days' fermentation, the highest TBARS value was observed in the control group at $1.93 \mathrm{mg} \mathrm{MDA} / \mathrm{kg}$ and sample with Lactoc. lactis M3 had the lowest TBARS value at $1.21 \mathrm{mg}$ 

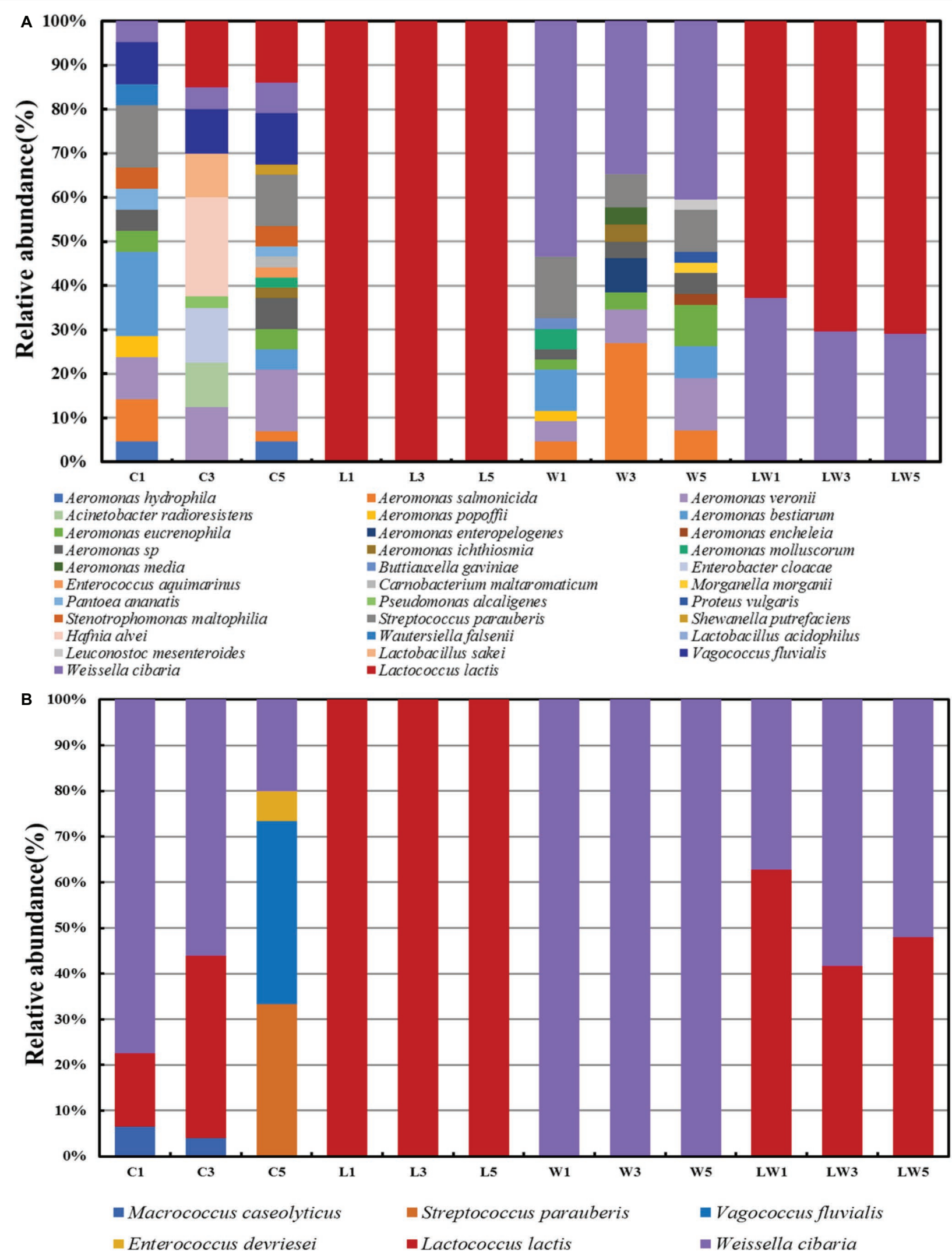

FIGURE 1 | Relative bacterial composition showing the bacterial succession during the fermentation process with and without starter culture obtained on plate count agar plates (A) and MRS plates (B). 
TABLE 2 | Changes of TVB-N, TBARS, and biogenic amines during the fermentation.

\begin{tabular}{|c|c|c|c|c|c|c|c|c|c|c|}
\hline \multirow{2}{*}{ Samples $^{A}$} & \multicolumn{3}{|c|}{ TVB-N (mg/100 g) } & \multicolumn{3}{|c|}{ TBARS (mg/kg) } & \multicolumn{4}{|c|}{ Biogenic amines (mg/kg) } \\
\hline & 1D & 3D & 5D & 1D & 3D & 5D & Histamine & Cadaverine & Tyramine & Putrescine \\
\hline C & $22.67 \pm 2.92^{\mathrm{a}}$ & $47.18 \pm 0.50^{c}$ & $49.49 \pm 0.99^{b}$ & $0.03 \pm 0.00^{\mathrm{a}}$ & $1.38 \pm 0.00^{\circ}$ & $1.98 \pm 0.02^{d}$ & $N^{a}$ & $3.08 \pm 1.27^{b}$ & $4.85 \pm 1.18^{c}$ & $25.19 \pm 4.16^{b}$ \\
\hline L & $22.90 \pm 0.99^{a}$ & $26.23 \pm 1.97^{a}$ & $34.17 \pm 0.99^{a}$ & $0.10 \pm 0.01^{b}$ & $1.35 \pm 0.00^{b}$ & $1.21 \pm 0.02^{a}$ & $N^{a}$ & $\mathrm{ND}^{\mathrm{a}}$ & $N^{a}$ & $5.46 \pm 2.57^{\mathrm{a}}$ \\
\hline W & $20.32 \pm 1.48^{a}$ & $35.82 \pm 0.50^{b}$ & $52.68 \pm 1.97^{b}$ & $0.72 \pm 0.04^{\mathrm{c}}$ & $1.12 \pm 0.00^{a}$ & $1.59 \pm 0.04^{b}$ & $N^{a}$ & $2.35 \pm 2.24^{b}$ & $2.72 \pm 0.52^{b}$ & $11.82 \pm 5.91^{\mathrm{a}}$ \\
\hline LW & $22.20 \pm 1.99^{a}$ & $26.36 \pm 0.00^{\mathrm{a}}$ & $47.49 \pm 2.98^{b}$ & $0.05 \pm 0.00^{\mathrm{a}}$ & $1.34 \pm 0.00^{b}$ & $1.79 \pm 0.10^{c}$ & $N^{a}$ & $0.53 \pm 0.52^{a}$ & $4.88 \pm 0.61^{\circ}$ & $9.90 \pm 1.99^{a}$ \\
\hline
\end{tabular}

Values with unlike superscript letters $(a, b)$ in the same column are significantly different ( $<<0.05) .{ }^{A} C, L, W$, and LW represent fish fermented without starter, with Lactoc. lactis M10, with W. cibaria M3, and with both of the bacteria.

MDA/kg. The TBARS values of the samples with and without starter cultures differed significantly $(p<0.05)$. It was reported that LAB had antioxidant effects on unsaturated fatty acids, and suppressed increase of TBARS in fermented fish (Zeng et al., 2013) and dry sausages (Sun et al., 2017). Therefore, it was reasonable that TBARS contents for products inoculated with Lactoc. lactis M10 and W. cibaria M3 could be definitely lower than that in the control group.

Biogenic amines, such as histamine, cadaverine, putrescine and tyramine, are potentially produced due to the growth of active microorganisms, acidification, and proteolysis that generates free amino acids during the fermentation process (Gardini et al., 2016). Changes in the concentrations of biogenic amines were monitored during fish fermentation and summarized in Table 2. Histamine contents were very low and were not detected in all the samples, while significant differences were found in putrescine, tyramine, and cadaverine between the control group and starter culture groups $(p<0.05)$. Compared with the control group, putrescine reduced to 21.6, 46.9, and $39.3 \%$ for the L, W and LW group, respectively. For cadaverine, the content in the starter cultures' were reduced to $0,76.3$ and $17.2 \%$ for each group as shown in Table 2. LAB starter culture has been commonly considered to suppress the growth of wild amine-producing bacteria, such as Enterobacteriaceae, which often contained high lysine- and ornithine-decarboxylase activities and are correlated with the production of cadaverine and putrescine (Bover-Cid et al., 2001; Durlu-Ozkaya et al., 2001). Therefore, the inoculation of Lactoc. lactis M3 or W. cibaria M3 may inhibit the non-starter LAB, such as Enterobacter cloacae in the control group, thus reducing the content of biogenic amines.

\section{Changes in Sensor Response Signals During the Fermentation}

Sensor responses in Chouguiyu among groups were measured during fermentation and shown in Figures $\mathbf{2 A - C}$ at days 1, 3, and 5 respectively. Sensor response values were observed to rise with the prolongation of fermentation time, indicating that the total volatile compounds increased gradually with the fermentation process. The sensor signals of $R(1), R(2), R(6), R(7), R(8)$, and $R(9)$ were stronger than others, which meant that these sensors were more sensitive and the correspondent substances, which were aromatics, nitrogen oxides, and sulfur-containing organics, were increased greatly during fermentation.
The radar map of L1 samples at the early stage of fermentation (Figure 2A) was the largest with an area of 8.30, while that of control sample was minimum of 5.00. Differences among other three groups were little. At the middle stage of fermentation, the LW3 samples' area of 11.69 was the largest in all samples. After five days' fermentation, the sensor profile of LW5 group was the most similar to the control group fermented for seven days (C7). The area of LW5 group was the largest in all starter culture groups, accounting for $86.74 \%$ of C7. The profile of W5 group, which was the second similar to the C7 group's, reached an area of 20.89 and accounted for $75.58 \%$ of the control group's. In contrast, L5 had the minimum area of 9.91. This result indicated that Lactoc. lactis M10 played an important role at the beginning of fermentation, while $W$. cibaria M3 contributed to produce flavor substances at the later fermentation period. When Lactoc. lactis M10 and W. cibaria M3 exist at the same time, it may promote the formation of flavor substances in the LW sample, which most resembled the $\mathrm{C} 7$ sample, and resulted in the fermentation time shortened by $29 \%$.

To reveal the relationship of the sample with or without starter cultures, the data of sensor response signals were processed by PCA. The first principal component explained $88.0 \%$ of the total variance, and the second principal component explained $1.54 \%$ of the total variance, indicating that the two principal components could fully reflect the characteristic information of the volatile substances in Chouguiyu with or without starters. At the early stage of fermentation, these four groups of samples were relatively concentrated and overlapped in their respective regions, indicating the differences between the electronic responses at the early stage of fermentation were not very significant. As the fermentation progressed, the differences were observed among the samples. At the end of fermentation, the samples of W5, LW5, and C7 were gathered separately from the other samples. Moreover, the overlap area of LW5 and C7 samples were larger than the others, indicating that the flavor of these two groups was the most similar. It could be inferred that $W$. cibaria $\mathrm{M} 3$ played an important role in the later stage of flavor formation, and co-inoculation of Lactoc. lactis M10 and $W$. cibaria M3 would promote the formation of flavor with significantly shortened time.

\section{Changes of Volatile Compounds Monitoring by GC-MS During the Fermentation}

The concentrations of volatile compounds in the samples with or without starters were analyzed by GC-MS and listed in Table S1. 

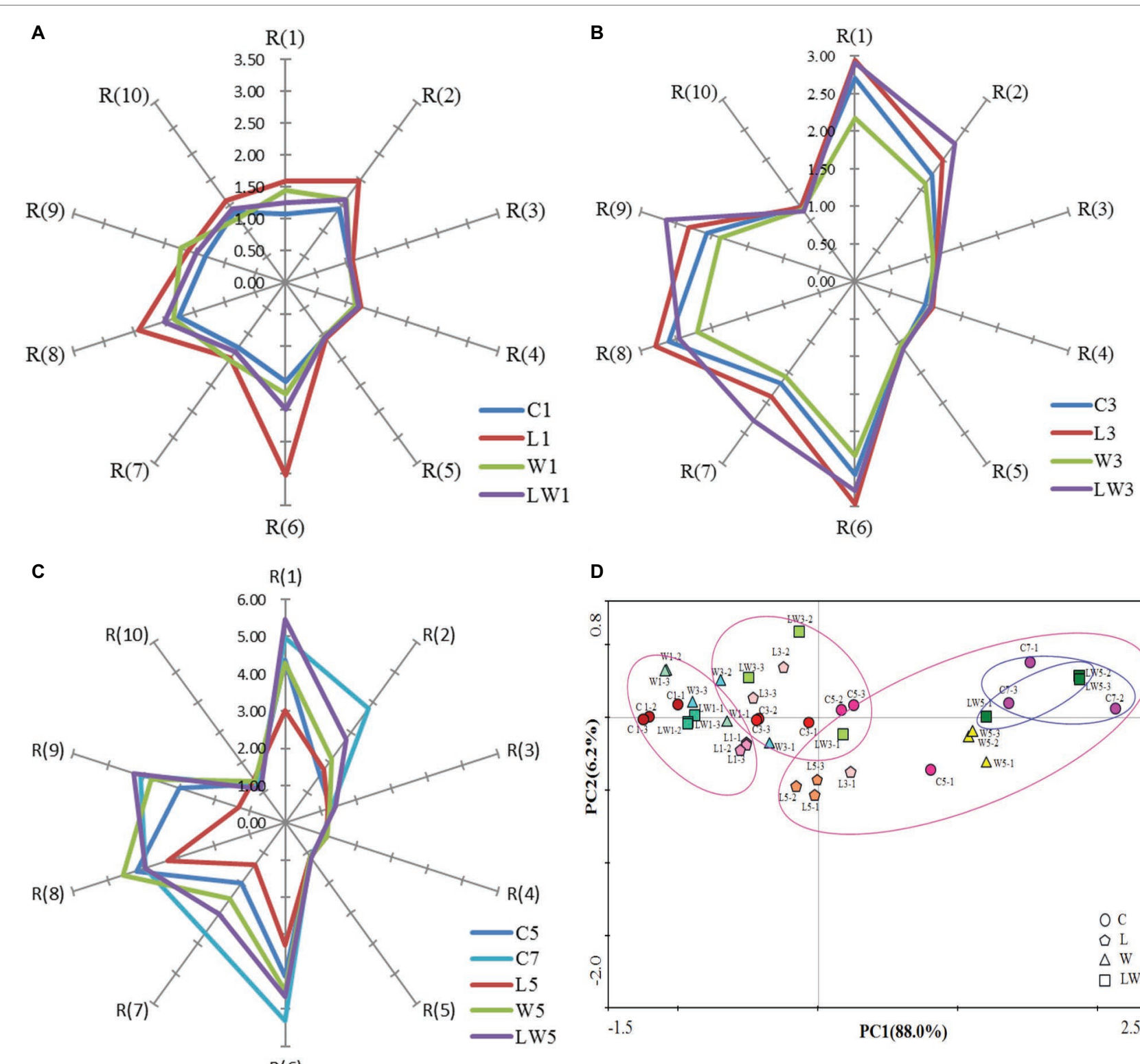

D

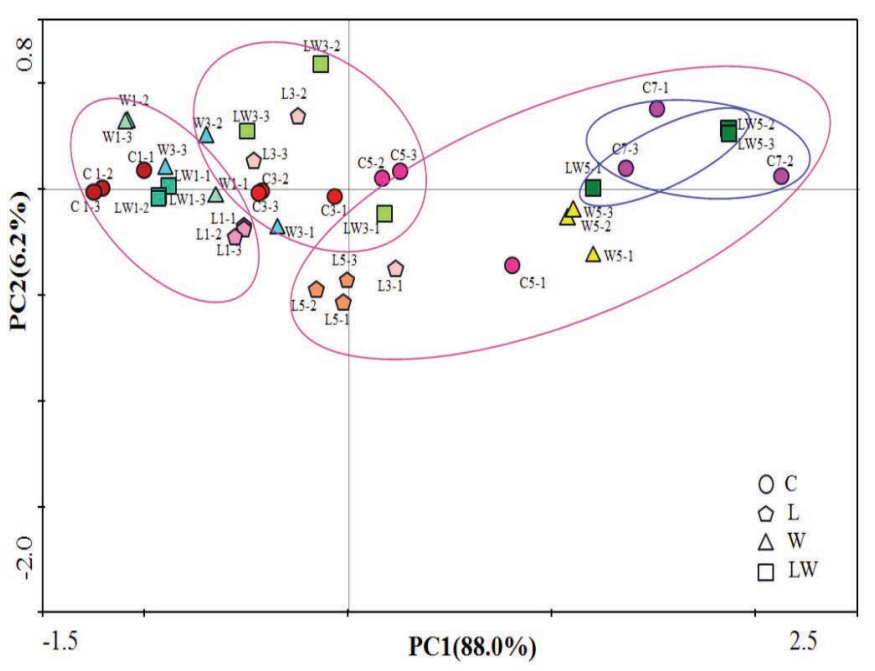

FIGURE 2 | E-nose analysis and PCA plot of samples inoculated with or without starter cultures. (A) E-nosc evaluation on days 1. (B) E-nose evaluation on days 3. (C) E-nose evaluation on days 5 and 7. (D) PCA results of E-nose profile evolution during the fermentation process. C, L, W and LW represented fish fermented without starter, with Lactoc. lactis M10, with W. cibaria M3 and with both of the bacteria.

A total of 43 volatile compounds were identified and quantified in 13 samples. Total contents of flavor substances were obviously increased (Figure 3) with the extension of fermentation time, especially for the proportion of aldehydes and aromatic substances. In the naturally fermented sample at the seventh day, volatile compounds were mainly abundant of $36.8 \%$ alcohols, $26.4 \%$ aldehydes, $18.3 \%$ aromatic compounds, and $8.5 \%$ ketones. What is more, increase of alcohols and acids was observed at the later stage of fermentation in both the C7 and LW5 groups, and volatile compound contents were significantly higher than that in the other groups. The volatile compound content in LW group at day 3 (6405.98 ng/100 g) could be comparable to that of natural fermentation at day $5(7074.23 \mathrm{ng} / 100 \mathrm{~g})$, and the content in LW group at day $5(11702.94 \mathrm{ng} / 100 \mathrm{~g})$ was even higher than that of natural fermentation at day $7(8055.79 \mathrm{ng} / 100 \mathrm{~g})$. This result indicated that the production of flavor substances was significantly enhanced by mixed inoculation, and the fermentation period was effectively shortened by $29 \%$.

Odor activity value (OAV) was defined as dividing the concentration of the odorant in the samples by the mean values of its estimated orthonasal threshold, and was widely used to identify the major odor-active compounds in food, such as fish sauce (Lapsongphon et al., 2015), wine (de Lerma et al., 2018), and cheese (Majcher and Jelen, 2011). Depending upon the volatile compounds' 


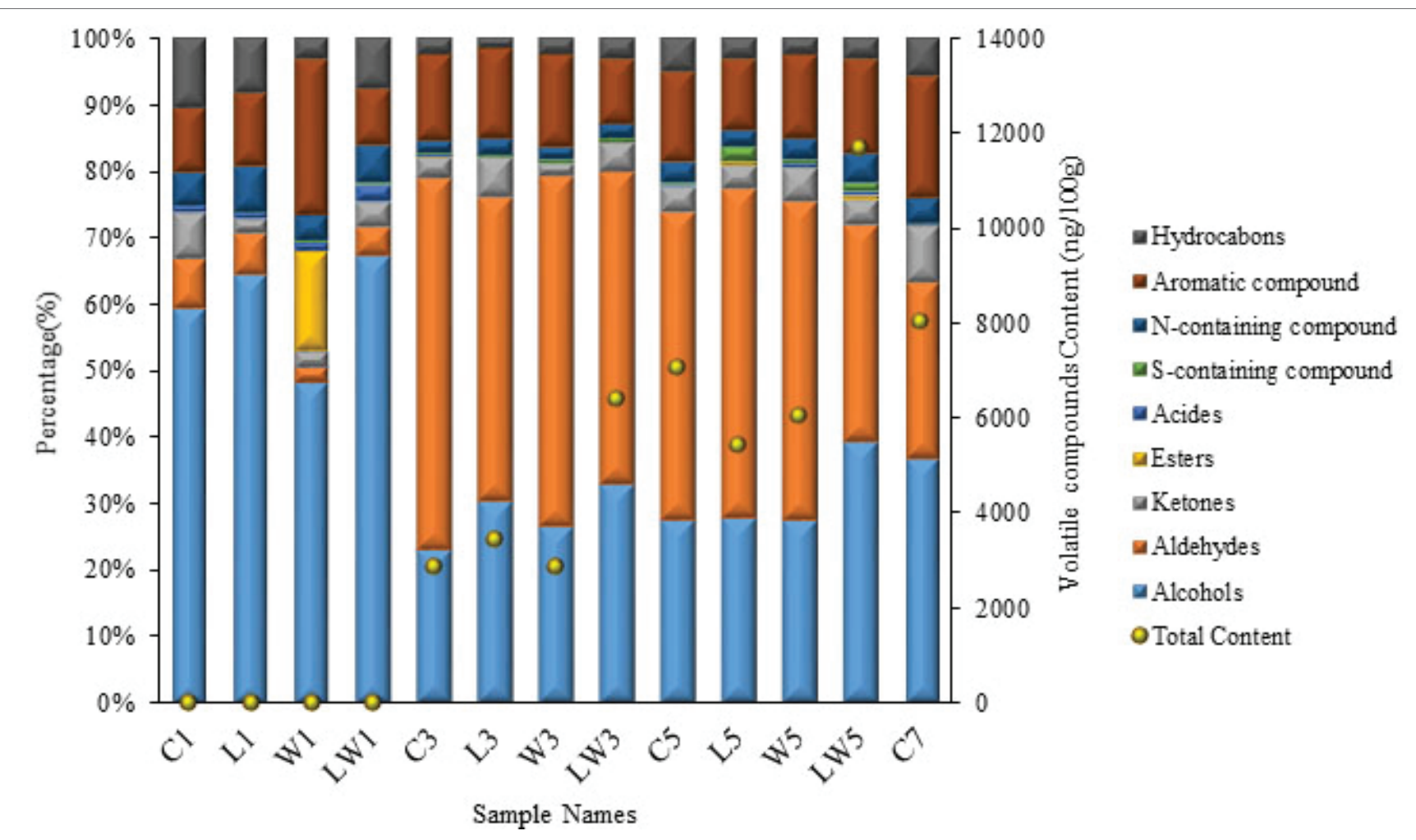

FIGURE 3 | Changes of different types and contents of volatile compounds in Chouguiyu with or without starter cultures during the fermentation process. C, L, W and LW represented fish fermented without starter, with Lactoc. lactis M10, with W. cibaria M3 and with both of the bacteria during the fermentation process. 5 and 7 represented that fish was fermented for 5 or 7 days.

thresholds and concentrations, a total of 11 compounds including 1-octen-3-ol, $\alpha$-terpineol, linalool, hexanal, heptanal, nonanal, oleic acid, indole, ethylbenzene, $\beta$-ocimene, and D-limonene, were found to have OAVs larger than 1. Consistent with our results, 1-octen-3-ol, hexanal, heptanal, nonanal, indole, and linalool were also recognized as major odor-active compounds of Chouguiyu in Li's report. In addition, these compounds showed a processdependent rising trend (Table S1), especially in the LW group. This result indicated that co-inoculation of Lactoc. lactis M10 and W. cibaria $\mathrm{M} 3$ had a positive effect for flavor production, especially for the aroma-active and key volatile compounds.

Aliphatic aldehydes, including hexanal, heptanal, and nonanal, were often found in fermented fish (Zeng et al., 2017), and were generally associated with unsaturated fatty acid autoxidation and enzymatic oxidation by starter microorganisms, such as Lactob. plantarum. These aldehydes could lead to a uniquely fatty, fishy, and nutty flavor in the Chouguiyu product due to their low odor thresholds. Indole has been reported to have an odor like putrid, musty, and floral on high dilutions. Liu et al. (2012) reported that indole was abundant in stinky tofu, a Chinese traditional soybean food. Similar to stinky tofu, indole was also abundant in the stinky fish product both in Li's (2013) report and in our study. Its concentration corresponded well with time, and was significantly greater in the LW group than other three groups at the same days. This result indicated that the inoculation of Lactoc. lactis M10 and $W$. cibaria M3 could promote the increase of indole content in the LW group. Another aroma-active compound, linalool, probably originated from spices in the process of manufacture, also showed an increasing trend with time, particularly in the LW group with the concentration of $1937.41 \mathrm{ng} / 100 \mathrm{~g}$ fish meat in LW5 sample. This substance from spices may dissolve in water and gradually penetrate into the fish body and give the product a uniquely botanical and citrus flavor to improve the overall flavor quality.

Hierarchical clustering analysis was performed with volatile compounds among different groups during the fermentation process. Samples appearing close in the tree are those that had a close proximity and the distance indicates the close relationship among samples. It could be deduced that flavor metabolites changed greatly at different stage. As shown in Figure 4, the volatile profiles of four samples in the initial stage of fermentation were obviously different from those in the late fermentation stages. Samples at the middle fermentation stage, which were C3, L3, W3, and LW3, clustered relatively close. Samples in the late fermentation stage were closer to naturally fermented product for 7 days. LW5 and C7 were classified in one cluster and showed similar metabolic activities. They were both characterized by high production of 1-octen-3-ol, $\alpha$-terpineol, 1-octanol, $\gamma$-terpineol, 2-methyl3-octanone, indole, 4-ethyl-o-xylene, anethole, pentamethyl, (1-methoxy-4-methyl-3-pentenyl)-benzene, and D-Limonene. The hierarchical clustering result was similar to that of PCA analysis based on E-nose measurement, and indicated that LW5 


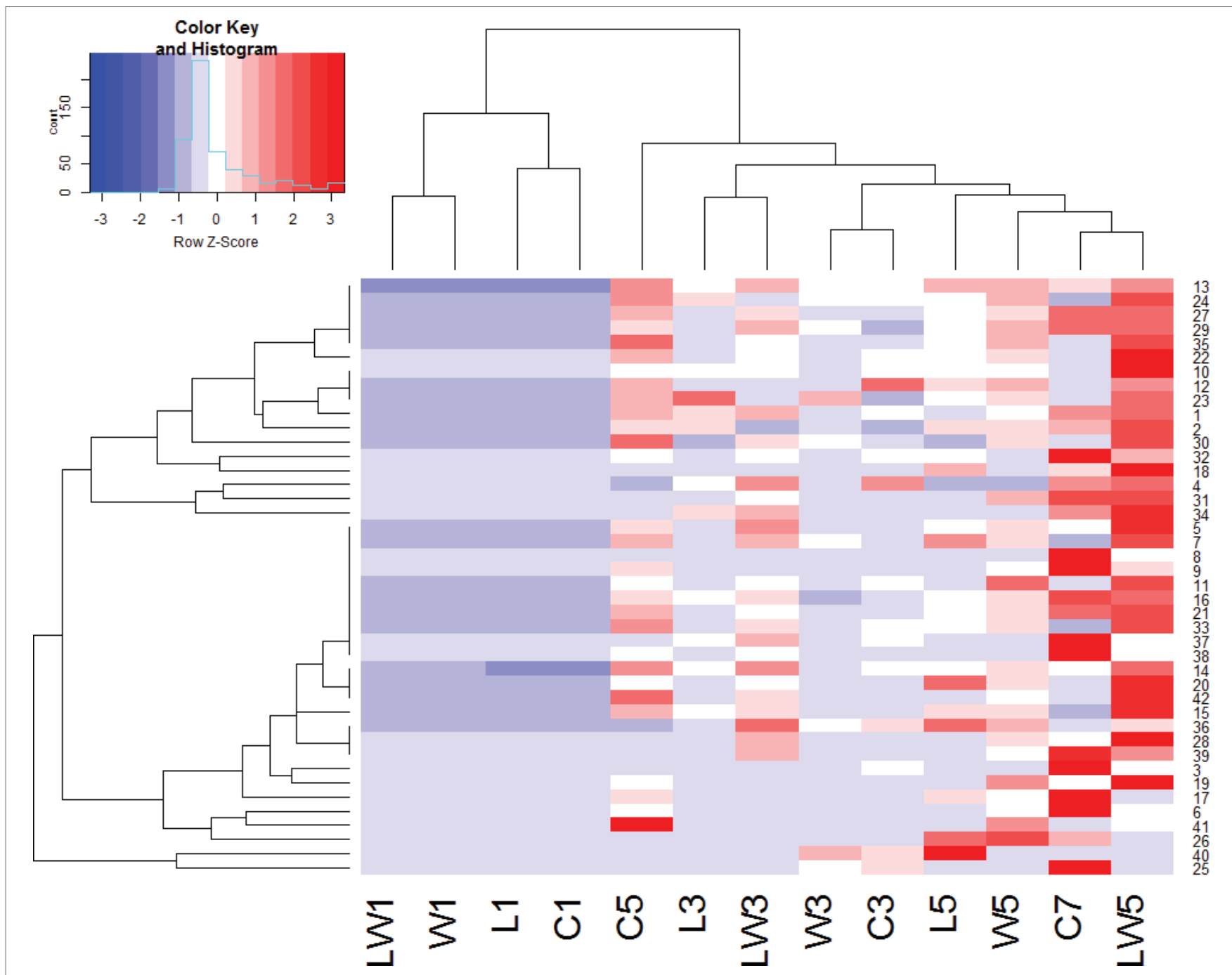

FIGURE 4 | Hierarchical clustering of volatile compounds in different fermentations. C, L, W and LW represented fish fermented without starter, with Lactoc. lactis M10, with $W$. cibaria M3 and with both of the bacteria during the fermentation process. 1-42 represented volatile compounds in Table S1.

and C7 were the most similar. Therefore, GC-MS results showed that inoculation of Lactoc. lactis M10 and W. cibaria M3 could promote the flavor formation in Chouguiyu products with time shortened.

\section{Sensory Evaluation}

In order to evaluate the acceptability of the product with or without starter cultures, sensory analysis, including appearance, aroma, taste, tactile texture, and overall acceptance, was carried out among the four groups (Table 3). There were no significant differences $(p<0.05)$ in the appearance and the texture of the products among four groups, indicating that inoculation of starter cultures did not affect appearance and texture. The activity of endogenous enzyme is the main factor contributing to the product's texture, such as the development of tenderness in beef (Cruzen et al., 2014). Therefore, it was easy to understand that microbial inoculation did not affect the texture of the product. For aroma and taste evaluation, the L group had the lowest score,
TABLE 3 | Sensory evaluation (mean panel score) of Chouguiyu by different starter cultures and control.

\begin{tabular}{lcccc}
\hline Attribute & C7 & L5 & W5 & LW5 \\
\hline Appearance & $7.15 \pm 0.42^{\mathrm{a}}$ & $7.15 \pm 0.79^{\mathrm{a}}$ & $7.21 \pm 0.62^{\mathrm{a}}$ & $7.21 \pm 0.63^{\mathrm{a}}$ \\
Aroma & $2.39 \pm 0.68^{\mathrm{b}}$ & $1.57 \pm 0.45^{\mathrm{a}}$ & $1.92 \pm 0.72^{\mathrm{ab}}$ & $2.39 \pm 0.68^{\mathrm{b}}$ \\
Taste & $6.42 \pm 0.64^{\mathrm{b}}$ & $5.28 \pm 0.80^{\mathrm{a}}$ & $5.67 \pm 0.50^{\mathrm{a}}$ & $6.54 \pm 0.69^{\mathrm{b}}$ \\
Tactile texture & $4.86 \pm 0.99^{\mathrm{a}}$ & $4.73 \pm 0.98^{\mathrm{a}}$ & $4.63 \pm 0.93^{\mathrm{a}}$ & $4.74 \pm 0.88^{\mathrm{a}}$ \\
Overall & $7.40 \pm 0.42^{\mathrm{b}}$ & $6.64 \pm 0.70^{\mathrm{a}}$ & $6.83 \pm 0.39^{\mathrm{a}}$ & $7.59 \pm 0.41^{\mathrm{b}}$ \\
acceptance & & & &
\end{tabular}

Values with different letters $(a, b)$ in the same row are significantly different $(p<0.05)$.

and was significantly different $(p<0.05)$ compared with the control group. The scores of LW group, both in aroma and taste, were the closest ones to C7 $(p<0.05)$. Zeng et al. (2017) reported that the quality of flavor compounds was remarkably improved by adding mixed starter cultures to Suanyu compared with that without inoculation. Xu et al. (2018) also reported that the 
formation of volatile compounds, which are essential for the distinct aroma in fermented fish, was mainly from microorganisms rather than endogenous enzymes. The overall acceptability in the LW group was higher than that in L or W group, and was the most similar to the naturally fermented group. This result indicated that co-inoculation of Lactoc. lactis M10 and W. cibaria M3 was able to promote the generation of flavor, improve the acceptability, and shorten the fermentation period at the same time.

\section{CONCLUSIONS}

Two indigenous LAB, Lactoc. lactis M10 and W. cibaria M3, were used to enhance the flavor production and quality properties of Chouguiyu product. Results showed that inoculation could accelerate the LAB dominant process and there might be some competitive relationship between Lactoc. lactis M10 and W. cibaria M3. LAB inoculation, especially in Lactoc. lactis M10, contributed to suppress the TVB-N, TBARS, and formation of biogenic amines in the final product. Flavor formation was firstly depended on Lactoc. lactis M10 and later on W. cibaria M3. Co-inoculation of these two could promote the flavor production and resulted in LW5 most resembling C7 in both E-nose and GC-MS analyses. Therefore, co-inoculation of Lactoc. lactis M10 and W. cibaria M3 in Chouguiyu could enhance the flavor formation, quality attributes as well as overall acceptance and shorten fermentation time.

\section{REFERENCES}

Bover-Cid, S., Izquierdo-Pulido, M., and Vidal-Carou, M. C. (2001). Changes in biogenic amine and polyamine contents in slightly fermented sausages manufactured with and without sugar. Meat Sci. 57,215-221. doi: 10.1016/s0309-1740(00)00096-6

Cobb, B. F., Alaniz, I., and Thompson, C. A. (1973). Biochemical and microbial studies on shrimp-volatile nitrogen and amino nitrogen analysis. J. Food Sci. 38, 431-436. doi: 10.1111/j.1365-2621.1973.tb01447.x

Cruzen, S. M., Paulin, P. V. R., Lonergan, S. M., and Huff-Lonergan, E. (2014). Postmortem proteolysis in three muscles from growing and mature beef cattle. Meat Sci. 96, 854-861. doi: 10.1016/j.meatsci.2013.09.021

da Cruz Pedrozo Miguel, M. G., de Castro Reis, L. V., Efraim, P., Santos, C., Lima, N., and Schwan, R. F. (2017). Cocoa fermentation: microbial identification by MALDITOF MS, and sensory evaluation of produced chocolate. LWT-Food Sci. Technol. 77, 362-369. doi: 10.1016/j.lwt.2016.11.076

Dai, Z., Li, Y., Wu, J., and Zhao, Q. (2013). Diversity of lactic acid bacteria during fermentation of a traditional Chinese fish product, Chouguiyu (stinky mandarinfish). J. Food Sci. 78, M1778-1783. doi: 10.1111/1750-3841.12289

de Almeida, M. A., Saldana, E., da Silva Pinto, J. S., Palacios, J., Contreras-Castillo, C. J., Sentandreu, M. A., and Fadda, S. G. (2018). A peptidomic approach of meat protein degradation in a low-sodium fermented sausage model using autochthonous starter cultures. Food Res. Int. 109, 368-379. doi: 10.1016/j.foodres.2018.04.042

de Lerma, N. L., Peinado, R. A., Puig-Pujol, A., Mauricio, J. C., Moreno, J., and GarciaMartinez, T. (2018). Influence of two yeast strains in free, bioimmobilized or immobilized with alginate forms on the aromatic profile of long aged sparkling wines. Food Chem. 250, 22-29. doi: 10.1016/j.foodchem.2018.01.036

Durlu-Ozkaya, F., Ayhan, K., and Vural, N. (2001). Biogenic amines produced by Enterobacteriaceae isolated from meat products. Meat Sci. 58, 163-166. doi: 10.1016/ s0309-1740(00)00144-3

El Hamzaoui, B., Laroche, M., Almeras, L., Berenger, J.-M., Raoult, D., and Parola, P. (2018). Detection of Bartonella spp. in fleas by MALDI-TOF MS. PLoS Negl. Trop. Dis. 12:e0006189. doi: 10.1371/journal.pntd.0006189

Essid, I., and Hassouna, M. (2013). Effect of inoculation of selected Staphylococcus xylosus and Lactobacillus plantarum strains on biochemical, microbiological and

\section{AUTHOR CONTRIBUTIONS}

$\mathrm{XL}, \mathrm{BZ}, \mathrm{SY}$, and XY conceived and planned the experiments. RB, SL, CJ, and HL performed the experiments. XL, BZ, and RB took the lead in writing the manuscript. All the above authors contributed to the interpretation of the results, provided critical feedback and helped shape the research, analysis and manuscript. $\mathrm{YZ}$ and XL revised the manuscript and proofed the final version of publication.

\section{FUNDING}

This study was funded by the National Key Research and Development Project (2016YFD0400404), the National Key Research and Development Project (2018YFD0400404), the High Level Talents Innovation and Entrepreneurship Project of Dalian (2017RQ045), and Natural Science Foundation of Liaoning Province (20170540057).

\section{SUPPLEMENTARY MATERIAL}

The Supplementary Material for this article can be found online at: https://www.frontiersin.org/articles/10.3389/fmicb.2018.03003/ full\#supplementary-material

textural characteristics of a Tunisian dry fermented sausage. Food Control 32, 707-714. doi: 10.1016/j.foodcont.2013.02.003

Fernandez-No, I. C., Bohme, K., Calo-Mata, P., Canas, B., Gallardo, J. M., and BarrosVelazquez, J. (2012). Isolation and characterization of Streptococcus parauberis from vacuum-packaging refrigerated seafood products. Food Microbiol. 30, 91-97. doi: 10.1016/j.fm.2011.10.012

Gao, P., Jiang, Q. X., Xu, Y. S., and Xia, W. S. (2018). Biosynthesis of acetate esters by dominate strains, isolated from Chinese traditional fermented fish (Suan yu). Food Chem. 244, 44-49. doi: 10.1016/j.foodchem.2017.10.007

Gardini, F., Ozogul, Y., Suzzi, G., Tabanelli, G., and Ozogul, F. (2016). Technological factors affecting biogenic amine content in foods: a review. Front. Microbiol. 7:18. doi: $10.3389 /$ fmicb. 2016.01218

Ho, V. T. T., Lo, R., Bansal, N., and Turner, M. S. (2018). Characterisation of Lactococcus lactis isolates from herbs, fruits and vegetables for use as biopreservatives against Listeria monocytogenes in cheese. Food Control 85, 472-483. doi: 10.1016/j. foodcont.2017.09.036

$\mathrm{Hu}, \mathrm{Y}$, Xia, W., and Ge, C. (2008). Characterization of fermented silver carp sausages inoculated with mixed starter culture. LWT-Food Sci. Technol. 41, 730-738. doi: 10.1016/j.lwt.2007.04.004

Ji, C., Zhang, J., Lin, X., Han, J., Dong, X., Yang, S., et al. (2017). Metaproteomic analysis of microbiota in the fermented fish, Siniperca chuatsi. LWT-Food Sci. Technol. 80, 479-484. doi: 10.1016/j.lwt.2017.03.022

John, L., Cornforth, D., Carpenter, C. E., Sorheim, O., Pettee, B. C., and Whittier, D. R. (2005). Color and thiobarbituric acid values of cooked top sirloin steaks packaged in modified atmospheres of $80 \%$ oxygen, or $0.4 \%$ carbon monoxide, or vacuum. Meat Sci. 69, 441-449. doi: 10.1016/j.meatsci.2004.08.013

Kargozari, M., Moini, S., Akhondzadeh Basti, A., Emam-Djomeh, Z., Gandomi, H., Revilla Martin, I., et al. (2014). Effect of autochthonous starter cultures isolated from Siahmazgi cheese on physicochemical, microbiological and volatile compound profiles and sensorial attributes of sucuk, a Turkish dry-fermented sausage. Meat Sci. 97, 104-114. doi: 10.1016/j.meatsci.2014.01.013

Lapsongphon, N., Yongsawatdigul, J., and Cadwallader, K. R. (2015). Identification and characterization of the aroma-impact components of Thai fish sauce. J. Agric. Food Chem. 63, 2628-2638. doi: 10.1021/jf5061248 
Lee, S. H., Jung, J. Y., and Jeon, C. O. (2014). Effects of temperature on microbial succession and metabolite change during saeu-jeot fermentation. Food Microbiol. 38, 16-25. doi: 10.1016/j.fm.2013.08.004

$\mathrm{Li}, \mathrm{C}$., Wu, J., Li, Y., and Dai, Z. (2013). Identification of the aroma compounds in stinky mandarin fish (Siniperca chuatsi) and comparison of volatiles during fermentation and storage. Int. J. Food Sci. Technol. 48, 2429-2437. doi: 10.1111/ijfs.12254

Li, S. W., Chen, Y. S., Lee, Y. S., Yang, C. H., Srionnual, S., Wu, H. C., et al. (2017). Comparative genomic analysis of bacteriocin-producing Weissella cibaria 110. Appl. Microbiol. Biotechnol. 101, 1227-1237. doi: 10.1007/s00253-016-8073-8

Liu, Y. P., Miao, Z. W., Guan, W., and Sun, B. G. (2012). Analysis of organic volatile flavor compounds in fermented stinky tofu using spme with different fiber coatings. Molecules 17, 3708-3722. doi: 10.3390/molecules17043708

Mah, J. H., Han, H. K., Oh, Y. J., Kim, M. G., and Hwang, H. J. (2002). Biogenic amines in Jeotkals, Korean salted and fermented fish products. Food Chem. 79, 239-243. doi: 10.1016/s0308-8146(02)00150-4

Majcher, M. A., and Jelen, H. H. (2011). Key odorants of Oscypek, a traditional polish Ewe's milk cheese. J. Agric. Food Chem. 59, 4932-4937. doi: 10.1021/jf2002602

Montes, M., Perez, M. J., and Nieto, T. P. (1999). Numerical taxonomy of gramnegative, facultative anaerobic bacteria isolated from skin of turbot (Scophthalmus maximus) and surrounding water. Syst. Appl. Microbiol. 22, 604-618. doi: 10.1016/ S0723-2020(99)80014-9

Park, Y., Lee, S., Hong, J., Kim, D., Moniruzzaman, M., and Bai, S. C. (2017). Use of probiotics to enhance growth, stimulate immunity and confer disease resistance to Aeromonas salmonicida in rainbow trout (Oncorhynchus mykiss). Aquac. Res. 48, 2672-2682. doi: 10.1111/are.13099

Romi, W., Ahmed, G., and Jeyaram, K. (2015). Three-phase succession of autochthonous lactic acid bacteria to reach a stable ecosystem within 7 days of natural bamboo shoot fermentation as revealed by different molecular approaches. Mol. Ecol. 24, 3372-3389. doi: 10.1111/mec.13237

Ruggirello, M., Giordano, M., Bertolino, M., Ferrocino, I., Cocolin, L., and Dolci, P. (2018). Study of Lactococcus lactis during advanced ripening stages of model cheeses characterized by GC-MS. Food Microbiol. 74, 132-142. doi: 10.1016/j.fm.2018.03.012

Saelao, S., Maneerat, S., Thongruck, K., Watthanasakphuban, N., Wiriyagulopas, S., Chobert, J. M., et al. (2018). Reduction of tyramine accumulation in Thai fermented shrimp (kung-som) by nisin Z-producing Lactococcus lactis KTH0-1S as starter culture. Food Control 90, 249-258. doi: 10.1016/j.foodcont.2018.03.003

Sun, F., Kong, B., Chen, Q., Han, Q., and Diao, X. (2017). N-nitrosoamine inhibition and quality preservation of Harbin dry sausages by inoculated with Lactobacillus pentosus, Lactobacillus curvatus and Lactobacillus sake. Food Control 73, 1514-1521. doi: 10.1016/j.foodcont.2016.11.018

Thierry, A., Pogacic, T., Weber, M., and Lortal, S. (2016). Biotechnology of lactic acid bacteria: novel applications, 2nd Edn. 314-340.
Woraprayote, W., Pumpuang, L., Tosukhowong, A., Roytrakul, S., Perez, R. H., Zendo, T., et al. (2015). Two putatively novel bacteriocins active against Gramnegative food borne pathogens produced by Weissella hellenica BCC 7293. Food Control 55, 176-184. doi: 10.1016/j.foodcont.2015.02.036

Xu, Y., Li, L., Regenstein, J. M., Gao, P., Zang, J., Xia, W., et al. (2018). The contribution of autochthonous microflora on free fatty acids release and flavor development in low-salt fermented fish. Food Chem. 256, 259-267. doi: 10.1016/j. foodchem.2018.02.142

Yang, P.-Z., Zhu, X.-X., Cao, L.-L., Cheng, J.-S., Zheng, Z., and Jiang, S.-T. (2016). Safety evaluation of Bacillus cereus isolated from smelly mandarin fish. J. Food Meas. Charact. 11, 726-735. doi: 10.1007/s11694-016-9442-9

Yang, S., He, Y., Yan, Y., Xie, N., Song, Y., Yan, X., et al. (2017). Textural properties of stinky mandarin fish (Siniperca chuatsi) during fermentation: effects of the state of moisture. Int. J. Food Prop. 20, 1530-1538. doi: 10.1080/10942912.2016.1233433

Yin, L. J., Pan, C. L., and Jiang, S. T. (2002). Effect of lactic acid bacterial fermentation on the characteristics of minced mackerel. J. Food Sci. 67, 786-792. doi: 10.1111/ j.1365-2621.2002.tb10677.x

Zeng, X., Chen, X., and Zhang, W. (2016). Characterization of the microbial flora from Suan Yu, a Chinese traditional low-salt fermented fish. J. Food Process. Preserv. 40, 1093-1103. doi: 10.1111/jfpp.12690

Zeng, X., Xia, W., Jiang, Q., Xu, Y., and Fan, J. (2017). Contribution of mixed starter cultures to flavor profile of suanyu-a traditional chinese low-salt fermented whole fish. J. Food Process. Preserv. 41:e13131. doi: 10.1111/jfpp.13131

Zeng, X., Xia, W., Jiang, Q., and Yang, F. (2013). Effect of autochthonous starter cultures on microbiological and physico-chemical characteristics of Suan yu, a traditional Chinese low salt fermented fish. Food Control 33, 344-351. doi: 10.1016/j. foodcont.2013.03.001

Zhang, Q., Lin, S., and Nie, X. (2013). Reduction of biogenic amine accumulation in silver carp sausage by an amine-negative Lactobacillus plantarum. Food Control 32, 496-500. doi: 10.1016/j.foodcont.2013.01.029

Conflict of Interest Statement: The authors declare that the research was conducted in the absence of any commercial or financial relationships that could be construed as a potential conflict of interest.

Copyright (c) 2018 Bao, Liu, Ji, Liang, Yang, Yan, Zhou, Lin and Zhu. This is an open-access article distributed under the terms of the Creative Commons Attribution License (CC BY). The use, distribution or reproduction in other forums is permitted, provided the original author(s) and the copyright owner(s) are credited and that the original publication in this journal is cited, in accordance with accepted academic practice. No use, distribution or reproduction is permitted which does not comply with these terms. 